\title{
Coriolis effects on rotating Hele-Shaw flows: A conformal-mapping approach
}

\author{
José A. Miranda* \\ Departamento de Física, Universidade Federal de Pernambuco, Recife, PE 50670-901, Brazil \\ Hermes Gadêlha ${ }^{\dagger}$ \\ Centre for Mathematical Biology, Mathematical Institute, University of Oxford, 24-29 St. Giles', Oxford OX1 3LB, United Kingdom \\ and The Capes Foundation, Ministry of Education of Brazil, Cx. Postal 365, Brasília, DF 70359-970, Brazil \\ Alan T. Dorsey \\ Department of Physics, University of Florida, P.O. Box 118440, Gainesville, Florida 32611-8440, USA
}

(Received 23 July 2010; published 6 December 2010)

\begin{abstract}
The zero surface tension fluid-fluid interface dynamics in a radial Hele-Shaw cell driven by both injection and rotation is studied by a conformal-mapping approach. The situation in which one of the fluids is inviscid and has negligible density is analyzed. When Coriolis force effects are ignored, exact solutions of the zero surface tension rotating Hele-Shaw problem with injection reveal suppression of cusp singularities for sufficiently high rotation rates. We study how the Coriolis force affects the time-dependent solutions of the problem, and the development of finite time singularities. By employing Richardson's harmonic moments approach we obtain conformal maps which describe the time evolution of the fluid boundary. Our results demonstrate that the inertial Coriolis contribution plays an important role in determining the time for cusp formation. Moreover, it introduces a phase drift that makes the evolving patterns rotate. The Coriolis force acts against centrifugal effects, promoting (inhibiting) cusp breakdown if the more viscous and dense fluid lies outside (inside) the interface. Despite the presence of Coriolis effects, the occurrence of finger bending events has not been detected in the exact solutions.
\end{abstract}

DOI: 10.1103/PhysRevE.82.066306

PACS number(s): 47.15.gp, 47.54.-r, 47.20.Ma, 47.15.km

\section{INTRODUCTION}

The classic Saffman-Taylor problem [1,2] serves as a paradigm for pattern formation in systems far from equilibrium. In the typical arrangement, a less viscous fluid pushes a more viscous one in the narrow gap of a Hele-Shaw cell, resulting in a viscosity-driven instability and complex interfacial patterns. The pattern morphology depends on control parameters such as the cell geometry, the fluid injection rate, and the interfacial surface tension. An even richer morphology results when the Hele-Shaw cell rotates about a perpendicular axis-inertial forces act upon the density difference between the fluids and can produce a density-driven instability. The interplay between these competing viscosity-and density-driven instabilities, studied in the early work of Schwartz [3] and Carrillo et al. [4], provides the motivation for the continued interest in the viscous fingering problem in rotating Hele-Shaw cells (see, for instance [5-10], and references therein).

While much of the early work on rotating Hele-Shaw cells focused on the role of the centrifugal force, more recently researchers have examined the role of the Coriolis force in rotating Hele-Shaw flows [11-16]. These works show that Coriolis effects have a significant influence on the dynamics and morphology of the resulting interfacial patterns. Linear stability analyses $[3,11,13]$ indicate that the Co-

\footnotetext{
*jme@df.ufpe.br

†gadelha@maths.ox.ac.uk

†atdorsey@ufl.edu
}

riolis force leads to a complex linear growth rate, so that growing fingers rotate with time. Numerical solutions $[15,16]$ show that Coriolis effects induce the formation of different pattern morphologies, where the tip of the fingering structures has the tendency to bend and drift from purely radial growth.

For zero surface tension, complex variable methods have been used to obtain exact solutions for the evolution of a fluid interface in a rotating Hele-Shaw cell [5-7] in the absence of Coriolis effects. Entov et al. [5] found classes of exact solutions for simply connected blobs and bubbles in a rotating cell. Their work has been extended to the case of a doubly connected fluid domain by Crowdy [7]. As in the nonrotating Hele-Shaw problem with zero surface tension [17-20], the exact solutions obtained in Refs. [5-7] may develop cusp singularities at finite time. However, when injection and the centrifugal force act simultaneously an interesting competition emerges [5,6] - depending upon the relative magnitudes between these effects, the centrifugal force could suppress the injection-induced cusp singularities. The present work extends and generalizes these previous studies by including the Coriolis force. In what follows we present exact solutions for the (zero surface tension) interface evolution in a rotating Hele-Shaw cell with injection and both centrifugal and Coriolis forces, and we study the effect of the Coriolis force on the formation of finite time cusp singularities.

We focus on zero surface tension situations in which one of the fluids is inviscid and has negligible density. Specifically, we consider two distinct cases, where (i) the central bubble is a less dense and less viscous fluid, and the one surrounding it is a viscous and more dense fluid; and where (ii) the central blob is that viscous more dense fluid, and a 


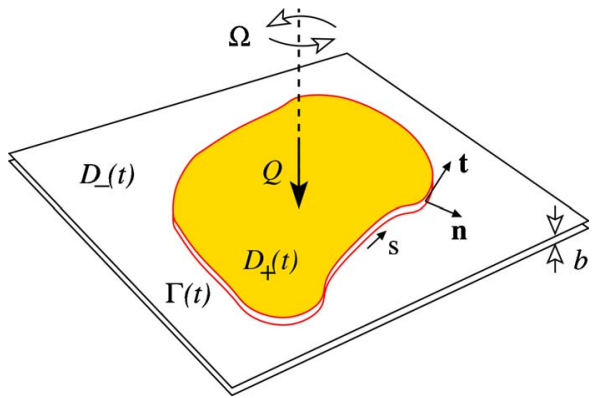

FIG. 1. (Color online) Schematic of the bubble geometry in the Hele-Shaw cell. The plates of the cell have spacing $b$ and rotate about a perpendicular axis with angular velocity $\Omega$. The inviscid bubble occupies the domain $D_{+}(t)$, the viscous fluid occupies $D_{-}(t)$, and the interface $\Gamma(t)$ separates the two domains. The unit vectors $\mathbf{n}$ and $\mathbf{t}$ are normal and tangent to the interface, and $s$ is an arc length parameter. The source of strength $Q$ is centered at the origin.

fluid of nearly zero density and viscosity surrounds it. This constitutes the high viscosity and high density contrast limit for which the conformal mapping formulation is a suitable scheme for providing useful analytical insights. Despite the relevance of an arbitrary viscosity contrast in affecting the emergence of other types of singularities (e.g., droplet pinchoff) in the nonzero surface tension problem [21,22], a conformal-mapping approach to this scenario would be far more complicated, and defies analytical treatment.

This paper is organized as follows. In Sec. II the problem is specified and the basic equations describing the system are presented. In Sec. III we extend the theory of harmonic moments originally proposed in Refs. $[5,17]$ by including the contribution of the Coriolis force into the Hele-Shaw problem under rotation and injection. By employing the formalism of Cauchy transforms, in Sec. IV we connect the moments to conformal maps, and generalize the exact solutions obtained in Refs. [5,6] by adding Coriolis effects. The major effects of the Coriolis force on the exact solutions and its influence on the formation of cusp singularities are illustrated in Sec. V. The possibility of avoiding cusps with rotation is reexamined in the light of the presence of the Coriolis contribution. Finally, Sec. VI contains a summary of our results along with comments regarding the implications of our results for nonzero surface tension boundary evolution $[3,15,16]$.

\section{GOVERNING EQUATIONS OF THE PROBLEM}

We begin by presenting a set of basic equations which describe the effectively two-dimensional (2D) viscous flow in a rotating Hele-Shaw cell. The geometry of the interface is shown schematically in Fig. 1. Consider the interface between a fluid of viscosity $\eta$ and density $\rho$, and another fluid of negligible viscosity and density in a Hele-Shaw cell of gap width $b$. The fluids are incompressible, and the cell rotates counterclockwise with constant angular velocity $\Omega$ around the $z$ axis (perpendicular to the cell plates). One fluid can be injected into the other through a hole at the center of rotation with a constant flow rate $Q$ (area per unit time). We will generally be interested in injection $(Q>0) ; Q<0$ corresponds to suction. The rotating coordinate system is defined in such a way that its origin is located at the center of the cell. The surface tension between the fluids is assumed to be zero.

The fluid flow in the rotating frame of reference is governed by the Navier-Stokes equation

$$
\begin{aligned}
\rho\left[\frac{\partial \mathbf{u}}{\partial t}+(\mathbf{u} \cdot \nabla) \mathbf{u}\right]= & -\boldsymbol{\nabla} p+\eta \nabla^{2} \mathbf{u}-\rho \boldsymbol{\Omega} \times(\boldsymbol{\Omega} \times \mathbf{r}) \\
& -2 \rho \boldsymbol{\Omega} \times \mathbf{u},
\end{aligned}
$$

and the continuity equation for incompressible fluids

$$
\boldsymbol{\nabla} \cdot \mathbf{u}=0,
$$

where $\mathbf{u}$ denotes the 3D fluid velocity, $p$ is the hydrodynamic pressure, $\boldsymbol{\Omega}=\Omega \mathbf{e}_{z}$ ( $\mathbf{e}_{z}$ is the unit vector along the $\mathrm{z}$ axis), and $\mathbf{r}$ is the displacement of a fluid element from the axis of rotation. The third term on the right hand side of Eq. (1) is the centrifugal force, and the fourth term is the Coriolis force.

As in the usual derivation of Darcy's law for flow in a Hele-Shaw cell from the Navier-Stokes equation, one simply ignores the standard inertial terms on the left hand side of Eq. (1), assumes a negligible pressure gradient in the $z$ direction across the cell thickness, and then averages over the $z$ direction to obtain a generalized Darcy's law $[11,13]$

$$
\mathbf{v}=-\frac{b^{2}}{12 \eta}\left[C \boldsymbol{\nabla} P-D \mathbf{e}_{z} \times \nabla P\right],
$$

where $\mathbf{v}$ represents the $2 \mathrm{D}$ fluid velocity (averaged over the gap), $P=p-\rho \Omega^{2} r^{2} / 2$ is the reduced pressure, and $C$ and $D$ are dimensionless functions of the Reynolds number $\operatorname{Re}=\rho \Omega b^{2} / 12 \eta$, given by

$$
\begin{gathered}
C=\frac{\sinh \sqrt{12 \mathrm{Re}}-\sin \sqrt{12 \mathrm{Re}}}{2 \operatorname{Re} \sqrt{12 \mathrm{Re}}[\cosh \sqrt{12 \mathrm{Re}}+\cos \sqrt{12 \mathrm{Re}}]}, \\
D=\frac{1}{2 \operatorname{Re}}\left\{1-\frac{\sinh \sqrt{12 \mathrm{Re}}+\sin \sqrt{12 \mathrm{Re}}}{\sqrt{12 \mathrm{Re}}[\cosh \sqrt{12 \mathrm{Re}}+\cos \sqrt{12 \mathrm{Re}}]}\right\} .
\end{gathered}
$$

When $\mathrm{Re} \ll 1$ we obtain $C=1+O\left(\mathrm{Re}^{2}\right)$ and $D=(12 / 5) \operatorname{Re}+O\left(\operatorname{Re}^{3}\right)$, and thus reproduce Darcy's law for a rotating Hele-Shaw cell without Coriolis forces [4]. It is important to note that the inertial effects give $C$ and $D$ a nontrivial dependence on Re, which is plotted in Fig. 2. From the incompressibility condition and Eq. (3), the reduced pressure is Laplacian,

$$
\nabla^{2} P=0
$$

Since the interface evolution is determined by solutions of Laplace's equation, the resulting patterns are examples of Laplacian growth.

The viscous fluid can be either inside or outside the interface; since both situations can be studied using the same set of equations, in what follows we focus on the case in which the viscous fluid is outside. This "bubble" geometry is illustrated in Fig. 1. The inviscid fluid occupies a domain $D_{+}(t)$, surrounded by the viscous fluid that occupies the exterior 


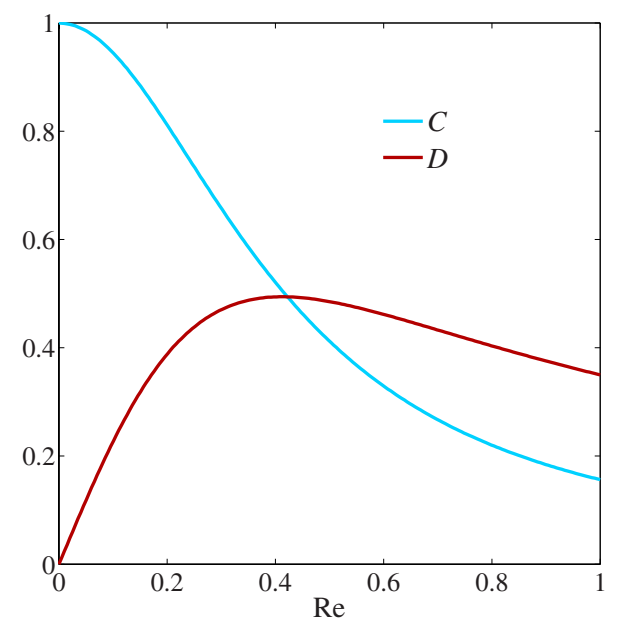

FIG. 2. (Color online) Plot of the expressions for the coefficients $C$ and $D$ in Eqs. (4) and (5), as a function of the Reynolds number Re. Note that the dependence on $\mathrm{Re}$ is due to the inclusion of the Coriolis force in the fluid equations of motion, as described in Refs. $[3,11]$.

domain $D_{-}(t)$. The two domains are separated by an interface $\Gamma(t)=\partial D_{+}(t)$. Without loss of generality we can assume that the absolute pressure $p$ inside $D_{+}$is zero, with the pressure in $D_{-}$given by Eq. (6). The motion of the interface is determined entirely by its normal velocity $V_{n}$ (the tangential velocity only leads to a relabeling of points on the interface), so we have

$$
V_{n}=\left.\mathbf{n} \cdot \mathbf{v}\right|_{\Gamma(t)},
$$

where $\mathbf{n}$ is a normal vector that is directed outward from the domain $D_{+}(t)$. Using Eq. (3), we obtain

$$
V_{n}=-\frac{b^{2}}{12 \eta}\left[C \partial_{n} P+D \partial_{s} P\right]
$$

with $\partial_{n} P$ the normal derivative of the reduced pressure, and $\partial_{s} P$ the tangential derivative of the reduced pressure (taken counterclockwise around $\Gamma(t))$. Under zero surface tension $p=0$ on the interface, so that the reduced pressure at $\Gamma(t)$ is

$$
\left.P\right|_{\Gamma(t)}=-\left.\frac{1}{2} \rho \Omega^{2} r^{2}\right|_{\Gamma(t)}
$$

Taking into account the existence of a single source at the origin of strength $Q$, the asymptotic behavior of the reduced pressure can be written as

$$
P \sim-\frac{Q}{2 \pi} \frac{12 \eta}{b^{2} C} \ln r, \quad r \rightarrow \infty .
$$

Equations (3)-(10) complete the description of our 2D Laplacian growth problem in a rotating Hele-Shaw cell with injection, including the effects of the Coriolis force.

\section{EVOLUTION OF THE HARMONIC MOMENTS}

The harmonic moments approach to interface dynamics was developed by Richardson [17], who investigated injection-driven, zero surface tension radial Hele-Shaw flow, in the case where the viscous fluid is inside the interface. The elegance of this approach is that the moments (defined below) are conserved during Laplacian growth-the nonlinear equations of motion for the interface are integrable. When combined with conformal mapping techniques the harmonic moments approach provides a compact and elegant treatment for a plethora of initial conditions and situations. Entov et al. [5] extended Richardson's approach to other situations, including centrifugally induced flows in rotating Hele-Shaw cells. Our purpose in this section is to further extend the approach to include the Coriolis force.

Following Refs. [17,5], we define the Cauchy transform of the domain $D_{-}$as

$$
h_{D}(w)=\frac{1}{\pi} \int_{D_{-}} \frac{d x d y}{w-z}=-\frac{1}{2 \pi i} \oint_{\partial D} \frac{\bar{z} d z}{w-z}=-\frac{1}{\pi} \sum_{k=1}^{\infty} M_{k}(t) w^{k-1},
$$

where $z=x+i y$, and $\bar{z}=x-i y$. The function $h_{D}(w)$ is the generating function of the moments $M_{k}$, which are given by

$$
M_{k}=\int_{D_{-}} z^{-k} d x d y=\frac{1}{2 i} \oint_{\Gamma(t)} z^{-k} \bar{z} d z, \quad k \geq 0 .
$$

These are the exterior moments, relevant when the viscous fluid is outside the interface, an arrangement referred to as the "bubble" case. To treat the "blob" case-when the viscous fluid is inside the interface-we would need the interior moments of $z^{k}(k \geq 0)$ [5]. In what follows we will focus on bubbles and the exterior moments with $k \geq 1$; the results for blobs can be derived in a similar fashion, and will be discussed at the end of this section.

Analogous to Fourier modes, each moment corresponds to a particular feature of the interface. The $k=0$ term gives the area exterior to the bubble (and its time derivative gives the rate at which fluid is being extracted from the cell), and the moments with $k \geq 1$ are proportional to the amplitude of a given modulation of the domain with exactly $k$ waves along the interface. By determining the time dependence of the harmonic moments one may construct exact solutions for the evolution of the interface $[5,17,23]$. To relate the dynamics of the moments to the interface evolution, we consider the time derivative of $M_{k}$ which follows from Eq. (12) as

$$
\frac{d M_{k}}{d t}=\oint_{\Gamma(t)} z^{-k} V_{n} d s
$$

with $V_{n}$ given by Eq. (8).

The first term that we need to calculate in Eq. (13) is

$$
\oint_{\Gamma(t)} z^{-k} \partial_{n} P d s=\oint_{\Gamma(t)}\left[z^{-k} \partial_{n} P-P \partial_{n} z^{-k}\right] d s+\oint_{\Gamma(t)} P \partial_{n} z^{-k} d s,
$$

where we have added and subtracted $P \partial_{n} z^{-k}$ from the integrand. Using Green's theorem, along with Eq. (9), Eq. (14) can be rewritten as 


$$
\begin{aligned}
\oint_{\Gamma(t)} z^{-k} \partial_{n} P d s= & \int\left[z^{-k} \nabla^{2} P-P \nabla^{2} z^{-k}\right] d a \\
& -\frac{1}{2} \rho \Omega^{2} \oint_{\Gamma(t)} r^{2} \partial_{n} z^{-k} d s,
\end{aligned}
$$

with $d a=d x d y$. The first integral on the right hand side of Eq. (15) is zero, since $\nabla^{2} P=0$ and $\nabla^{2} z^{-k}=0\left(z^{-k}\right.$ is analytic away from the origin). For the remaining integral in Eq. (15), using the divergence theorem we find

$$
\begin{aligned}
\oint_{\Gamma(t)} r^{2} \partial_{n} z^{-k} d s & =\oint_{\Gamma(t)} r^{2} \mathbf{n} \cdot \boldsymbol{\nabla} z^{-k} d s \\
& =\int \boldsymbol{\nabla} \cdot\left(r^{2} \boldsymbol{\nabla} z^{-k}\right) d a \\
& =2 \int \mathbf{r} \cdot \boldsymbol{\nabla} z^{-k} d a \\
& =-2 k \int z^{-k} d a
\end{aligned}
$$

Therefore, the integral on the left hand side of Eq. (14) can be finally written as

$$
\oint_{\Gamma(t)} z^{-k} \partial_{n} P d s=\rho \Omega^{2} k M_{k} .
$$

Next, we evaluate the term in Eq. (13) which involves the tangential derivative of the reduced pressure

$$
\oint_{\Gamma(t)} z^{-k} \partial_{s} P d s=-\frac{1}{2} \rho \Omega^{2} \oint_{\Gamma(t)} z^{-k} \partial_{s} r^{2} d s,
$$

where we have used Eq. (9), along with $\partial_{s} p=0$ on the interface (in the absence of surface tension). Note that this tangential derivative term is new, and is due to the Coriolis force. Using $\partial_{s} r^{2}=2 \mathbf{r} \cdot \mathbf{t}$, where $\mathbf{t}=\partial_{s} \mathbf{r}=\mathbf{e}_{z} \times \mathbf{n}$ is the tangent vector to the curve $\Gamma(t)$, we find $\partial_{s} r^{2}=2\left(\mathbf{r} \times \mathbf{e}_{z}\right) \cdot \mathbf{n}$. Using the divergence theorem once again, we have

$$
\begin{aligned}
\oint_{\Gamma(t)} z^{-k} \partial_{s} r^{2} d s & =2 \oint_{\Gamma(t)} z^{-k}\left(\mathbf{r} \times \mathbf{e}_{z}\right) \cdot \mathbf{n} d s \\
& =2 \int \boldsymbol{\nabla} \cdot\left[z^{-k}\left(\mathbf{r} \times \mathbf{e}_{z}\right)\right] d a \\
& =2 \int\left(-y \partial_{x}+x \partial_{y}\right) z^{-k} d a \\
& =-2 i k \int z^{-k} d a
\end{aligned}
$$

Therefore, the integral in Eq. (18) is

$$
\oint_{\Gamma(t)} z^{-k} \partial_{s} P d s=i \rho \Omega^{2} k M_{k}
$$

Combining Eqs. (8), (13), (17), and (20), we obtain an expression for the time derivative of the exterior harmonic moments

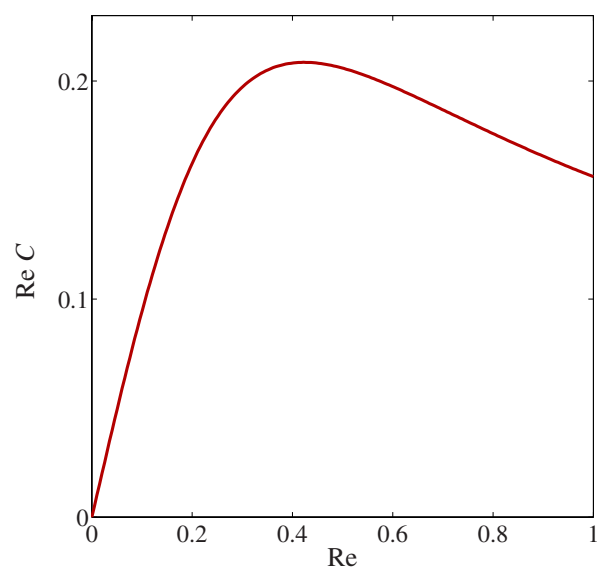

FIG. 3. (Color online) Plot of $\operatorname{Re} C$ as a function of the Reynolds number Re. This quantity controls the real part of the growth rate for the harmonic moments, at fixed angular velocity. There is a pronounced nonmonotonic dependence on the Reynolds number $\mathrm{Re}$, with a maximum at $\mathrm{Re}=0.42$.

$$
\frac{d M_{k}}{d t}=-(\lambda+i \omega) k M_{k} \quad(k \geq 1),
$$

where

$$
\lambda=\Omega \operatorname{Re} C=\Omega^{*} C, \quad \omega=\Omega \operatorname{Re} D=\Omega^{*} D,
$$

and $\Omega^{*}=\rho \Omega^{2} b^{2} / 12 \eta$. Equation (21) can be readily integrated leading to

$$
M_{k}(t)=M_{k}(0) e^{-(\lambda+i \omega) k t} \quad(k \geq 1) .
$$

For the zeroth moment, we have

$$
M_{0}(t)=M_{0}(0)+Q t .
$$

Equations (21)-(24) constitute one of the central results of this work, yielding the explicit time dependence of the harmonic moments on including injection, centrifugal, and Coriolis effects. Note that when Coriolis effects are neglected, these results agree with Eq. (4.1) of Entov et al. [5].

In the presence of rotation the harmonic moments are no longer conserved; they do, however, decouple from one another and can be easily integrated. Assuming that the interface is regular (no singularities develop), at large time all of the exterior moments tend to zero and the bubble eventually becomes circular. The quantity $\lambda$ is the real part of the growth rate for the harmonic moments; it reduces to the parameter $\Omega^{*}$ defined by Magdaleno et al. [6] when Coriolis effects are neglected $(C=1$ and $D=0)$. At fixed $\Omega$ the growth rate is controlled by the dimensionless quantity $\operatorname{Re} C$, which is plotted in Fig. 3. An interesting feature of this growth rate is the nonmonotonic dependence on the Reynolds number $\mathrm{Re}$, the direct result of the Coriolis force. As expected, the centrifugal force plays a stabilizing role when the viscous fluid is outside the interface. However, the interface could develop a singularity in finite time before the perturbation can be stabilized by centrifugal effects, so there will in general be a competition between injection and rotation. The 
Coriolis force also produces an imaginary part in the growth rate and the moments acquire a complex phase factor, resulting in rotation of the bubbles.

It should be noted that the calculation of the interior moments (the "blob" case) follows along the same lines, resulting in analogous expressions. In this case the negative sign appearing in Eqs. (21) and (23) should be replaced by a plus sign. The interior moments grow exponentially, so that the centrifugal contribution is destabilizing. Conversely, since the viscous fluid is the one injected against the inviscid outer fluid, now injection has a stabilizing role. This scenario also leads to the development of cusps in finite time [5,6]. As in the bubble case, one issue which still merits further investigation is the influence of the Coriolis force on the formation of cusp singularities, which will be discussed in Secs. IV and V.

\section{CONNECTION BETWEEN HARMONIC MOMENTS AND CONFORMAL MAPS FOR BUBBLES}

We can make further progress using conformal mapping techniques. Let $z=f(\zeta)$ map the exterior of the curve $\Gamma(t)$ in the $z$ plane to the interior of the unit circle in the $\zeta$ plane. To make the connection with Magdaleno et al. [6], we start by considering the map

$$
f(\zeta)=a_{-1}(t) \zeta^{-1}+a_{0}(t)+a_{N}(t) \zeta^{N},
$$

where in general $a_{-1}, a_{0}$, and $a_{N}$ can be complex [take $\vartheta=-1$ in Eq. (5) of Magdaleno et al. for the case of a viscous fluid outside of $\Gamma$; our $a_{-1}$ corresponds to $a_{0}$ of Magdaleno, and our $a_{N}$ corresponds to Magdaleno's $a_{n+1}$ ]. The case $N=1$ corresponds to an ellipse, and $N=2$ corresponds to a three-cusped hypotrochoid. Note that the parameter $a_{0}$ shifts the center of mass of the pattern away from the origin. If we ignore these shifts, then in general map [Eq. (25)] produces a pattern that has a $(N+1)$-fold symmetry.

Once $a_{-1}, a_{0}$, and $a_{N}$ have been determined, the curve $\Gamma$ in the $z$ plane is obtained by writing $\zeta=e^{i \theta}$, and then separating the real and imaginary parts of $f$; we then have the parametric equations for the curve in the form $x(\theta, t)=\operatorname{Re}(f)$, $y(\theta, t)=\operatorname{Im}(f)$. Our next task is to calculate the equations that describe the time evolution of the conformal map coefficients.

\section{A. Method 1: Brute force calculation of the moments}

Using the contour integral representation of the moments in Eq. (12), we use the conformal map $z=f(\zeta)$ to transform the integral into a contour integral around the unit circle in the $\zeta$ plane

$$
M_{k}=\frac{1}{2 i} \oint_{\Gamma(t)} z^{-k} \bar{z} d z=\frac{1}{2 i} \oint_{|\zeta|=1} f^{*}(1 / \zeta)[f(\zeta)]^{-k} f^{\prime}(\zeta) d \zeta,
$$

where $f^{\prime} \equiv d f / d \zeta$ and $f^{*}(1 / \zeta) \equiv \overline{f(1 / \bar{\zeta})}$. To see how this works, first consider the zeroth moment (which is the area inside the curve $\Gamma$ in the $z$-plane) for the map [Eq. (25)] above [see Eq. (4.4) of Entov et al. [5]],

$$
\begin{aligned}
M_{0} & =\frac{1}{2 i} \oint_{|\zeta|=1} \bar{f} f^{\prime} d \zeta \\
& =\frac{1}{2 i} \oint_{|\zeta|=1}\left[\bar{a}_{-1} \zeta+\bar{a}_{0}+\bar{a}_{N} \zeta^{-N}\right]\left[-a_{-1} \zeta^{2}+N a_{N} \zeta^{N-1}\right] d \zeta \\
& =\pi\left[-\left|a_{-1}\right|^{2}+N\left|a_{N}\right|^{2}\right],
\end{aligned}
$$

where we have used

$$
f^{*}(1 / \zeta) \equiv \overline{f(1 / \bar{\zeta})}=\bar{a}_{-1} \zeta+\bar{a}_{0}+\bar{a}_{N} \zeta^{-N},
$$

for $\zeta=e^{i \theta}$ on the unit circle. The quantity above is minus the area inside $\Gamma$, and for constant injection rate $Q$ is given by $M_{0}=-\left(Q_{0} t+\pi k_{0}\right)$, and $k_{0}$ a constant, we obtain

$$
\left|a_{-1}\right|^{2}-N\left|a_{N}\right|^{2}=\frac{Q}{\pi} t+k_{0},
$$

which is exactly Eq. (9) of Magdaleno et al. [6].

In principle one could carry out this same calculation for higher moments. In practice this becomes quite cumbersome. Instead, we will move on to Method 2.

\section{B. Method 2: Compare singularities of conformal map and generating function}

The trick here is to use Eq. (2.8) of Entov et al. [5]. The arguments are subtle and rely on analytic continuation arguments for the moment generating function. The result is that there exists a function $\phi(\zeta)$, defined as

$$
\phi(\zeta)=f^{*}(1 / \zeta)-h_{D}[f(\zeta)],
$$

that is analytic in the domain $D$, where the generating function $h_{D}[f(\zeta)]$ has been given in Eq. (11). Since the functions $f^{*}(1 / \zeta)$ and $h_{D}[f(\zeta)]$ have the same singularities in $D$, analyticity of $\phi$ means that we will need to choose the parameters in the conformal map so as to cancel the nonanalytic terms. Suppose that the function $h_{D}(w)$ contains just a single term; i.e., $M_{k}(0)=0$ for $k \neq n$. Let us also set $a_{0}=0$, so that from now on in this work we will be considering patterns whose center of mass remains at the origin. Then

$$
h_{D}(w)=-\frac{M_{N+1}(0)}{\pi} e^{-(N+1)(\lambda+i \omega) t} w^{N} .
$$

Substituting Eq. (25) into this expression, we have

$$
\begin{aligned}
h_{D}(f(\zeta))= & -\frac{M_{N+1}(0)}{\pi} e^{-(N+1)(\lambda+i \omega) t} \times\left[a_{-1} \zeta^{-1}+a_{N} \zeta^{N}\right]^{N} \\
= & -\frac{M_{N+1}(0)}{\pi} e^{-(N+1)(\lambda+i \omega) t} a_{-1}^{N} \zeta^{-N} \\
& + \text { terms analytic in } \zeta .
\end{aligned}
$$

Combining this with Eq. (28), we have

$$
\begin{aligned}
\phi(\zeta)= & {\left[\bar{a}_{N}+\frac{M_{N+1}(0)}{\pi} e^{-(N+1)(\lambda+i \omega) t} a_{-1}^{N}\right] \zeta^{-N} } \\
& + \text { terms analytic in } \zeta .
\end{aligned}
$$

To eliminate the nonanalytic term $\zeta^{-N}$, we need to choose the constants $a_{-1}$ and $a_{N}$ such that 


$$
\frac{a_{-1}^{N}}{\bar{a}_{N}}=k_{N} e^{(N+1)(\lambda+i \omega) t},
$$

where $k_{N}=-\pi / M_{N+1}(0)$ is a constant that depends upon the initial conditions; we can choose it to be real without loss of generality. This expression is the generalization of Eq. (10) of Magdaleno et al. [6] to the case of a nonzero Coriolis force (recall that $\lambda$ is simply $\Omega^{*}$ in their notation).

In Ref. [6] the parameters $a_{-1}$ and $a_{N}$ were real, but here they are complex. To further simplify, write

$$
a_{-1}=\left|a_{-1}\right| e^{i \alpha_{-1}}, \quad a_{N}=\left|a_{N}\right| e^{i \alpha_{N}} .
$$

Substituting into Eq. (34), we have

$$
\frac{\left|a_{-1}\right|^{N}}{\left|a_{N}\right|} e^{i\left[N \alpha_{-1}+\alpha_{N}-(N+1) \omega t\right]}=k_{N} e^{(N+1) \lambda t} .
$$

Since the right hand side of Eq. (36) is real, we need to choose the quantity in the brackets on the left hand side to be an integer multiple of $2 \pi$; we can choose this integer to be zero without loss of generality, so that the phases $\alpha_{-1}$ and $\alpha_{N}$ satisfy

$$
N \alpha_{-1}+\alpha_{N}-(N+1) \omega t=0 .
$$

So, our final solution is given by Eq. (29), along with

$$
\frac{\left|a_{-1}\right|^{N}}{\left|a_{N}\right|}=k_{N} e^{(N+1) \lambda t},
$$

which together will determine the amplitudes $\left|a_{-1}\right|$ and $\left|a_{N}\right|$ as a function of time, and Eq. (37), which will determine the phases. Our Eqs. (29), (37), and (38) generalize the results obtained in Refs. [5,6] to include the Coriolis force. There is one possible problem-there is only one equation for the phases, and there are two phases. However, this apparent inconsistency is a non-issue as we will show below.

Once the coefficients in the conformal map have been determined by using Eqs. (29), (37), and (38), we can find a parametric solution for the interface $\Gamma$ by using $z=f(\zeta)$, $\zeta=e^{i \theta}$, and separating out real and imaginary parts. The result is

$$
\begin{aligned}
& x=\left|a_{-1}\right| \cos \left(\theta-\alpha_{-1}\right)+\left|a_{N}\right| \cos \left(N \theta+\alpha_{N}\right), \\
& y=-\left|a_{-1}\right| \sin \left(\theta-\alpha_{-1}\right)+\left|a_{N}\right| \sin \left(N \theta+\alpha_{N}\right) .
\end{aligned}
$$

If we introduce a new variable $\phi=\theta-\alpha_{-1}$, and use Eq. (37) for the phases, we find

$$
\begin{aligned}
& x=\left|a_{-1}\right| \cos (\phi)+\left|a_{N}\right| \cos [N \phi+(N+1) \omega t], \\
& y=-\left|a_{-1}\right| \sin (\phi)+\left|a_{N}\right| \sin [N \phi+(N+1) \omega t],
\end{aligned}
$$

so that the phases have dropped out entirely.

\section{ROLE OF THE CORIOLIS FORCE: ILLUSTRATIVE EXAMPLES}

\section{A. Bubble case}

First, we examine the bubble case in which the more viscous and more dense fluid is outside the interface. To make apparent the effects of the Coriolis force on the appearance of finite time cusp singularities, we consider a domain with a $(N+1)$-fold symmetry. By taking $N=2$ and using Eqs. (29) and (38) the coupled algebraic equations for the amplitudes are

$$
\begin{gathered}
\left|a_{-1}\right|^{2}-2\left|a_{2}\right|^{2}=\frac{Q}{\pi} t+k_{0}, \\
\frac{\left|a_{-1}\right|^{2}}{\left|a_{2}\right|}=k_{2} e^{3 \lambda t},
\end{gathered}
$$

where the constants $k_{0}$ and $k_{2}$ are determined by the initial conditions. After some algebra, and setting $\varepsilon=2\left[\left|a_{2}(0)\right| /\left|a_{-1}(0)\right|\right]$ and $\left|a_{-1}(0)\right|=1$ we find

$$
\begin{gathered}
\left|a_{2}\right|=\frac{e^{3 \lambda t}}{2 \varepsilon}-\sqrt{\frac{e^{6 \lambda t}}{4 \varepsilon^{2}}-\frac{1}{2}\left[\frac{Q}{\pi} t-\frac{\varepsilon^{2}}{2}+1\right],} \\
\left|a_{-1}\right|=\sqrt{\frac{2 e^{3 \lambda t}\left|a_{2}\right|}{\varepsilon}} .
\end{gathered}
$$

Note that a singularity will occur when the argument of the square root in Eq. (45) becomes negative. This happens at a critical time $t_{c}$ such that

$$
e^{6 \lambda t_{c}}=2 \varepsilon^{2}\left[\frac{Q}{\pi} t_{c}-\frac{\varepsilon^{2}}{2}+1\right] .
$$

In fact, this expression for $N=2$ can be generalized for any integer $N \geq 2$ yielding

$$
e^{2(N+1 / N-1) \lambda t_{c}}=\left(\frac{N}{N-1}\right) \varepsilon^{2 /(N-1)}\left[\frac{Q}{\pi} t_{c}-\frac{\varepsilon^{2}}{N}+1\right],
$$

where $\varepsilon=N\left[\left|a_{N}(0)\right| /\left|a_{-1}(0)\right|\right]$ and $\left|a_{-1}(0)\right|=1$. Recall that $\lambda=\Omega^{*} C$, and that $C$ is a function of the Reynolds number as given by Eq. (4). For $\mathrm{Re}=0$ it is known that, for sufficiently small injection rate $Q$ Eq. (47) may never be fulfilled, which means that the centrifugal force has stabilized the interface and removed any singularities [5,6]. On the other hand, for sufficiently large $Q$ we will have a singularity, leading to a cusped threefold hypotrochoid.

In order to investigate the role played by Coriolis effects on the growth of the interface, and on the development of cusp singularities, we use Eqs. (41), (42), (45), and (46) to visualize the time evolution of the rotating bubble. We stress that, for the results presented in this paper, the evolution is shown as seen in the rotating frame of reference in which the Hele-Shaw cell appears stationary. To make some contact with practical situations $[2,4,23]$, throughout this section we use cgs units, so that time is measured in seconds $(\mathrm{s}), \Omega^{*}$ has units of $\mathrm{s}^{-1}$, and $Q$ has units of $\mathrm{cm}^{2} / \mathrm{s}$. The conformal map amplitudes are measured in centimeters. On the other hand, Re and $\varepsilon$ are dimensionless.

Figure 4 depicts the time evolution of the fluid-fluid interface, when the inviscid fluid is injected into the viscous one: when Coriolis effects are omitted [Figs. 4(a)-4(c)], and included [Figs. 4(d)-4(f)]. We take $Q=\pi \mathrm{cm}^{2} / \mathrm{s}, \varepsilon=0.5$ and present successive contours differing by a fixed time interval. In Figs. 4(a)-4(c) the Reynolds number is set to zero, and the 

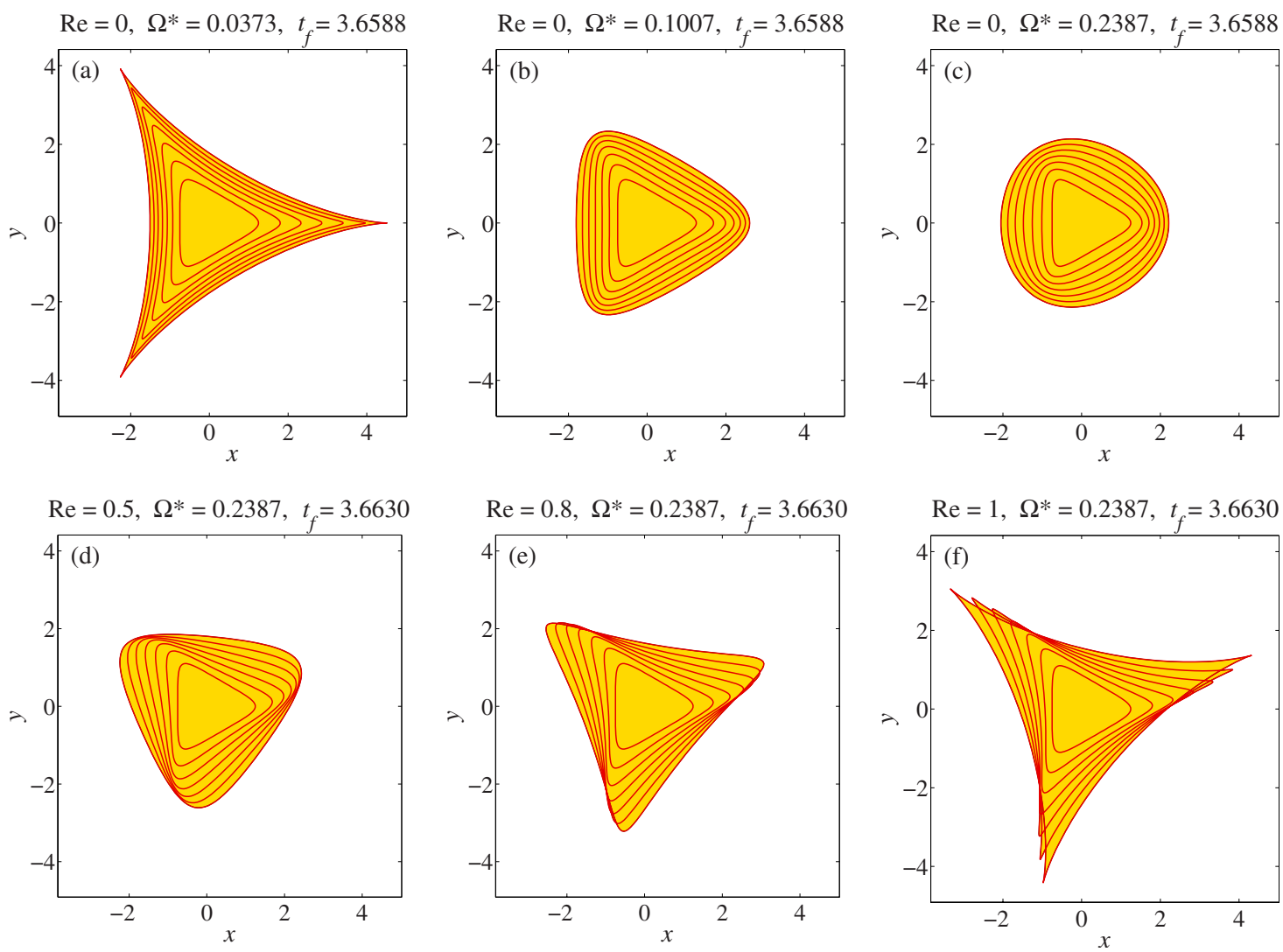

FIG. 4. (Color online) Illustrative gallery of possible behaviors for the time evolution of the rotating bubble interface. The upper row depicts the situations in which the Coriolis effects are neglected $(\mathrm{Re}=0)$. The rotation parameter $\Omega^{*}$ is increased from left to right, and the final times $t_{f}$ are all equal in Figs. 4(a)-4(c). The centrifugal force tends to stabilize the interface and inhibits cusp formation. The action of the Coriolis force is shown in the lower row [Figs. 4(d)-4(f)]. The Reynolds number is increased, while $\Omega^{*}$ is kept unchanged, and equal to its value in Fig. 4(c). Coriolis force destabilizes the flow, introducing a counterclockwise phase drift and favoring the reappearance of cusp singularities. In Fig. 4(f) cusps arise at time $t_{f}=3.6630$ s. Note that this is the final time considered in Figs. 4(d) and $4(\mathrm{e})$. $\Omega^{*}$ has units of $\mathrm{s}^{-1}$, and $Q$ has units of $\mathrm{cm}^{2} / \mathrm{s}$.

parameter $\Omega^{*}$ increases from left to right. At lower $\Omega^{*}$ $\left(\Omega^{*} \leq \Omega_{c}^{*}=0.0373 \mathrm{~s}^{-1}\right)$ rotation cannot prevent cusp formation, which occurs at time $t_{c}=3.6588 \mathrm{~s}$. Figures $4(\mathrm{~b})$ and $4(\mathrm{c})$ are plotted by considering $\left(\Omega^{*}>\Omega_{c}^{*}\right)$, and final time $t_{f}=t_{c}$. It is evident that increasingly larger values of $\Omega^{*}$ tend to inhibit cusp formation, and also lead to a stronger stabilization of the evolving interface. This is in agreement with the main result of Ref. [6] which states that (for $\operatorname{Re}=0$ ) there exists a critical rotation rate above which cusps are eliminated.

The zero Reynolds number scenario is considerably modified when Coriolis force effects are included. This can be seen in Figs. 4(d)-4(f) which plot the evolving interface by taking the same $\Omega^{*}$ as the one used in Fig. 4(c) $\left(\Omega^{*}=0.2387 \mathrm{~s}^{-1}\right)$, assuming a final time $t_{f}=3.6630 \mathrm{~s}$, but now considering increasingly larger values of Re: $0.5,0.8$, and 1 . We emphasize that in the laboratory it is relatively straightforward to achieve a Reynolds number of order 1 with conventional fluids and small plate spacings [24]. Observing Fig. 4(d), we readily notice that the Coriolis force acts to destabilize the interface [in the sense that the final contour is not circular as in Fig. 4(c)], and the patterns rotate in the counterclockwise direction. These basic features are also present in Fig. 4(e), where even more deformed inter- faces arise, exhibiting a phase shift as they grow. However, an interesting feature is seen in Fig. 4(f): for a sufficiently large Reynolds number $(\mathrm{Re}=1)$ one observes the rebirth of cusp singularities at time $t_{f}=3.6630 \mathrm{~s}$. Therefore, although in Fig. 4(f) $\Omega^{*}$ is larger than $\Omega_{c}^{*}$ for $\mathrm{Re}=0$ [used in Fig. 4(a)], the cusps are not at all suppressed. This unique effect is exclusively due to the Coriolis force, and is a consequence of the nonmonotonic behavior of the growth rate $\operatorname{Re} C$ plotted in Fig. 3. It is worth noting that, in contrast to what is observed in numerical simulations of the rotating Hele-Shaw problem with nonzero surface tension $[3,15,16]$, the growing structures presented in Figs. 4(d)-4(f) drift, but do not bend at their tips.

A useful way of organizing all the information contained in Figs. 4(a)-4(f) is to make a "phase diagram" in the $\Omega^{*}-$ Re parameter space as shown in Fig. 5. The dividing curve separates the stable and unstable regions of the interface development. Such a curve is obtained by numerically solving Eq. (47). For a given Reynolds number, along this separating curve one precisely gets the critical rotation rate $\Omega_{c}^{*}$ above which cusp singularities are stabilized. The diamonds plotted in Fig. 5 identify the various situations examined in Figs. 4(a)-4(f). If $\operatorname{Re}=0$ and $\Omega^{*}=\Omega_{c}^{*}[(\mathrm{a})]$ cusps still emerge. If $\mathrm{Re}$ is kept fixed, and $\Omega^{*}>\Omega_{c}^{*}$ one crosses to the 


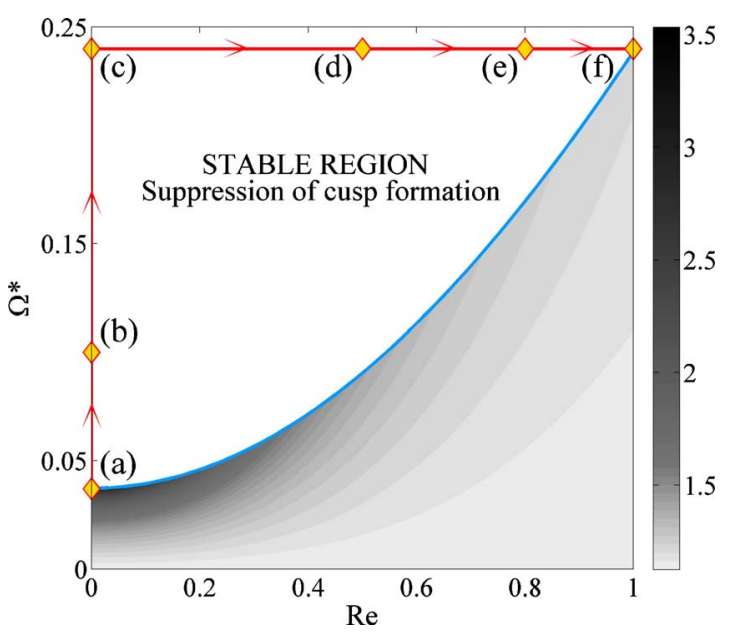

FIG. 5. (Color online) Phase diagram showing the critical curve at which $\Omega^{*}=\Omega_{c}^{*}$, and the stable zone where cusps are suppressed. The shaded regions below the critical curve indicate the times for cusp formation $t_{c}$ (labeled by the gray scale on right hand side of the graph). The diamonds show the values chosen for the time evolution plots shown in Figs. 4(a)-4(f). $\Omega^{*}$ has units of $\mathrm{s}^{-1}$, and $t$ is given in seconds.

stable region so that cusps are no longer present [(b)]. Further increase in the value of $\Omega^{*}$ leads to additional stabilization and the successive interfaces tend to a nearly circular shape [(c)]. However, it is not guaranteed that cusps are eliminated, and depending on the strength of the Coriolis effects, cusps may reappear. This can be conveniently achieved by tuning the Reynolds number, as observed in Figs. 5(c)-5(f). By increasing Re one eventually crosses the dividing critical curve, and cusp singularities appear. Therefore, when the viscous and more dense fluid is outside, contrary to the centrifugal force, the Coriolis effects act to destabilize the interface. Also notice that for fixed $\Omega^{*}$ in the unstable zone, the critical time for cusp generation decreases for larger values of the Reynolds number. From Figs. 4 and 5 we see that the consideration of the Coriolis contribution enriches the cusp singularity formation problem in HeleShaw flow by introducing a more subtle interplay of injection, centrifugal and Coriolis effects.

\section{B. Blob case}

Here we turn to the case in which the viscous and more dense fluid is inside the interface (blob case). The calculation of the equations of the conformal map amplitudes and phases for the case of a blob follows along the same line of the corresponding derivation for the bubble (Sec. IV). Consider the map $[5,6,25]$

$$
f(\zeta)=a_{1}(t) \zeta+a_{N}(t) \zeta^{N},
$$

which produces patterns that have a $(N-1)$-fold symmetry, where $N \geq 3$. Under such circumstances, we obtain expressions similar to Eqs. (29), (37), and (38). The equations for the amplitudes are now written as

$$
\left|a_{1}\right|^{2}+N\left|a_{N}\right|^{2}=\frac{Q}{\pi} t+k_{0},
$$

$$
\left|a_{1}\right|^{N}\left|a_{N}\right|=k_{N} e^{(N-1) \lambda t},
$$

where the constants $k_{0}$ and $k_{N}$ are determined by the initial conditions. Moreover, the phases are now related as

$$
N \alpha_{1}-\alpha_{N}+(N-1) \omega t=0 .
$$

To investigate the action of the Coriolis force on the time evolution of a rotating blob, we focus on a specific example for which $N=4$. Unfortunately, closed-form expressions for the amplitudes $\left|a_{1}(t)\right|$ and $\left|a_{4}(t)\right|$ cannot be determined analytically. The evolution of the interface is then described by the numerical solution of the following algebraic equations:

$$
\begin{gathered}
\left|a_{1}\right|^{10}-\left[\frac{Q}{\pi} t+\frac{\varepsilon^{2}}{4}+1\right]\left|a_{1}\right|^{8}+\frac{\varepsilon^{2}}{4} e^{6 \lambda t}=0, \\
\left|a_{4}\right|=\frac{\varepsilon}{4\left|a_{1}\right|^{4}} e^{3 \lambda t},
\end{gathered}
$$

where $\varepsilon=4\left[\left|a_{4}(0)\right| /\left|a_{1}(0)\right|\right]$ and $\left|a_{1}(0)\right|=1$. As in the bubble case, depending on the interplay of injection, centrifugal, and Coriolis contributions a finite time singularity may occur. In fact, it emerges at a critical time $t=t_{c}$ given by

$$
e^{6 \lambda t_{c} / 5}=\frac{4}{5 \varepsilon^{2 / 5}}\left[\frac{Q}{\pi} t_{c}+\frac{\varepsilon^{2}}{4}+1\right],
$$

leading to a pattern known as a three-cusped epicycloid. This specific result for $N=4$ can be generalized for any integer $N \geq 3$ leading to

$$
e^{2(N-1 / N+1) \lambda t_{c}}=\left(\frac{N}{N+1}\right) \frac{1}{\varepsilon^{2 /(N+1)}}\left[\frac{Q}{\pi} t_{c}+\frac{\varepsilon^{2}}{N}+1\right],
$$

where we take $\varepsilon=N\left(\left|a_{N}(0)\right| /\left|a_{1}(0)\right|\right)$ and $\left|a_{1}(0)\right|=1$. In agreement to Refs. [5,6] injection of the more viscous and dense fluid with a bounded injection rate cannot fully stabilize the flow. Therefore, formation of cusps can be delayed, but not avoided.

Figure 6(a) depicts the time evolution of an initially circular blob which is pumped with injection rate $Q=\pi \mathrm{cm}^{2} / \mathrm{s}$ into a rotating Hele-Shaw cell, when Coriolis effects are neglected $(\operatorname{Re}=0)$. We take $\Omega^{*}=0.25 \mathrm{~s}^{-1}$, and $\varepsilon=0.05$. Despite the stabilizing role played by injection, the interface is centrifugally destabilized, so that its boundary retreats, and eventually cusp singularities arise at a time $t_{c}=t_{f}=11.7307 \mathrm{~s}$. Figure 6(b) compares the final interface obtained in Fig. 6(a) for $\mathrm{Re}=0$, with the corresponding interfaces generated at $t=11.7307 \mathrm{~s}$ for two finite values of the Reynolds number: $\mathrm{Re}=0.05$ and $\mathrm{Re}=0.1$. In contrast to the bubble case, it is evident from Fig. 6(b) that the Coriolis effects tend to stabilize the flow, delaying the development of cusp singularities. We have verified that in the end, even if $\operatorname{Re} \neq 0$, cusps will ultimately emerge, but for larger critical times than those obtained when $\mathrm{Re}=0$. Even though the $\mathrm{Co}-$ riolis force is not capable of eliminating the formation of cusps, it is interesting that it acts against the destabilizing centrifugal effects. Moreover, as opposed to the bubble case, the patterns rotate in the clockwise direction. By changing the location (inside or outside the interface) of the more viscous and more dense fluid one modifies the direction of 

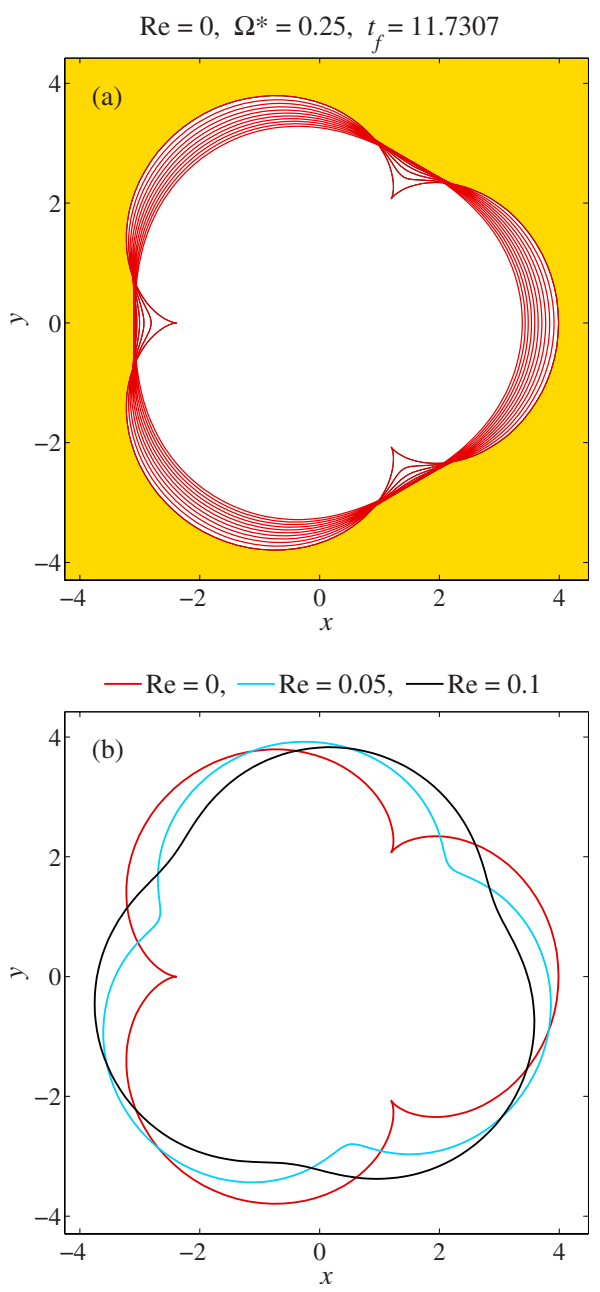

FIG. 6. (Color online) (a) Time evolution of the interface for the blob case when Coriolis effects are ignored $(\mathrm{Re}=0)$, for $Q=\pi \mathrm{cm}^{2} / \mathrm{s}, \Omega^{*}=0.25 \mathrm{~s}^{-1}$, and $\varepsilon=0.05$. Equally spaced time steps are used. Cusps arise at the critical time $t_{c}=t_{f}=11.7307$ s. (b) Overlaid interfaces at time $t_{f}=11.7307 \mathrm{~s}$, for three different values of the Reynolds number Re: 0, 0.05, and 0.1.

phase drift. Notice that, as in the bubble case, bending has not been detected.

Some other aspects are illustrated in Fig. 7 which plots the critical time for cusp singularity formation as a function of the Reynolds number, for three different values of the injection rate $Q: 0, \pi$, and $2 \pi$. The values of the parameters $\Omega^{*}$ and $\varepsilon$ are the same as those used in Fig. 6. Regardless of the value of $\operatorname{Re}$, the dashed curve $(Q=0)$ always lies below the two solid curves $(Q>0)$. As expected, this indicates that larger $Q$ leads to an enhanced stabilization of the system with respect to cusp formation. Another piece of information can be extracted by comparing the distance between the dashed curve (which considers centrifugal effects, but that neglects Coriolis force, and assumes $Q=0$ ), and any of the two solid curves (for which centrifugal, Coriolis, and injection effects are considered). So, for a finite fixed injection rate we observe that the distance separating the dashed and solid curves significantly increases as Re is ramped up. This observation expresses the increased effectiveness of the

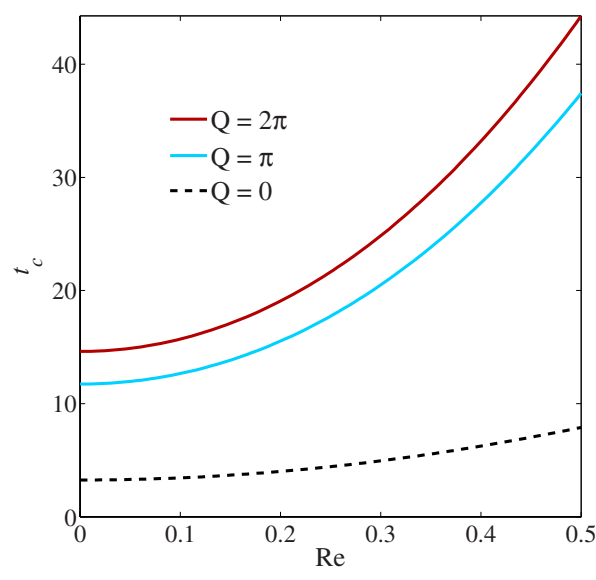

FIG. 7. (Color online) Time for cusp formation (measured in seconds) as a function of the Reynolds number Re for three different values of the injection rate $Q$. Here $\Omega^{*}=0.25 \mathrm{~s}^{-1}$, and $\varepsilon=0.05$. The curves are obtained by using Eq. (55).

Coriolis force in delaying the occurrence of cusp breakdown when it acts in conjunction with injection. On the other hand, we also notice that the distance between the two solids curves remains nearly unchanged as $\mathrm{Re}$ is varied. In this sense, changing $Q$ does not result in a dramatic modification in the cusp formation time $t_{c}$. In this scenario, the major contributor for more significant changes in $t_{c}$ is indeed the Coriolis force.

\section{CONCLUSIONS}

The existing conformal mapping treatment of zero surface tension Hele-Shaw flows neglects effects due to inertia. However, recent investigations of the rotating Hele-Shaw problem with nonzero surface tension [11-16] have shown that inertial corrections associated with the Coriolis force play an important role in determining the shape and stability of the fluid interface. Indeed, Refs. [24,26-29] introduced inertial corrections into the Saffman-Taylor problem with finite surface tension in rectangular Hele-Shaw cells. In the same spirit, we have examined the influence of the Coriolis force on the time evolution and development of cusp singularities in the rotating Hele-Shaw problem with injection, with zero surface tension.

Using a generalized Darcy's law which accounts for inertial Coriolis effects, we employ Richardson's harmonic moments approach to obtain the conformal maps that describe the time evolution of the interface. Our results indicate that the Coriolis contribution introduces two major effects: it induces a phase drift making the patterns to rotate as time passes, and significantly affects the time at which cusp singularities appear. The cusp formation time is governed by a balance between viscous forces, centrifugal driving, and rotational inertia, and cusps that were inhibited at low Reynolds numbers may reappear at larger Reynolds numbers. Finally, we have found that the Coriolis force always opposes centrifugal effects, so that the occurrence of cusp singularities is either favored or inhibited depending on the location of the more viscous and more dense fluid. 
It is worthwhile to note that the phase drift exhibited by our zero surface tension solutions is in agreement with a similar behavior detected in numerical simulations of the problem with nonzero surface tension. On the other hand, the ubiquitous finger bending phenomena observed in Refs. $[3,15,16]$ do not exist in our solutions. One might speculate that, with appropriate regularization (surface tension, viscous stresses, wetting effects, etc.) the high curvature portions of the cusped patterns would round out, and eventually bend as seen in the physically realizable situations. Our hope is that our results may stimulate future developments on this challenging topic.

\section{ACKNOWLEDGMENTS}

J.A.M. thanks CNPq (Brazilian Research Council) for financial support of this research through the program "Instituto Nacional de Ciência e Tecnologia de Fluidos Complexos (INCT-FCx)," and also through the CNPq/FAPESQ Pronex program. H.G. wishes to thank CAPES (Brazilian sponsor) for financial support through Grant No. BEX 4676/06-8. A.T.D. acknowledges support from NSF Grant No. DMR0705690, and from the Aspen Center for Physics, where part of this work was completed.
[1] P. G. Saffman and G. I. Taylor, Proc. R. Soc. London, Ser. A 245, 312 (1958).

[2] L. Paterson, J. Fluid Mech. 113, 513 (1981).

[3] L. W. Schwartz, Phys. Fluids A 1, 167 (1989).

[4] Ll. Carrillo, F. X. Magdaleno, J. Casademunt, and J. Ortín, Phys. Rev. E 54, 6260 (1996).

[5] V. M. Entov, P. I. Etingof, and D. Ya. Kleinbock, Eur. J. Appl. Math. 6, 399 (1995).

[6] F. X. Magdaleno, A. Rocco, and J. Casademunt, Phys. Rev. E 62, R5887 (2000).

[7] D. Crowdy, Q. Appl. Math. 60, 11 (2002); SIAM (Soc. Ind. Appl. Math.) J. Appl. Math. 62, 945 (2002).

[8] C.-Y. Chen and S.-W. Wang, Fluid Dyn. Res. 30, 315 (2002).

[9] E. Álvarez-Lacalle, J. Ortín, and J. Casademunt, Phys. Rev. Lett. 92, 054501 (2004).

[10] J. Casademunt, Chaos 14, 809 (2004).

[11] S. L. Waters and L. J. Cummings, Phys. Fluids 17, 048101 (2005).

[12] S. L. Waters, L. J. Cummings, K. M. Shakesheff, and F. R. A. J. Rose, IMA J. Math. Appl. Med. Biol. 23, 311 (2006).

[13] H. Gadêlha, N. Brito, and J. A. Miranda, Phys. Rev. E 75, 016305 (2007).

[14] A. Abidate, S. Aniss, O. Caballina, and M. Souhar, Phys. Rev. E 75, 046307 (2007).
[15] C.-Y. Chen, C.-W. Huang, H. Gadêlha, and J. A. Miranda, Phys. Rev. E 78, 016306 (2008).

[16] E. Alvarez-Lacalle, H. Gadêlha, and J. A. Miranda, Phys. Rev. E 78, 026305 (2008).

[17] S. Richardson, J. Fluid Mech. 56, 609 (1972).

[18] B. Shraiman and D. Bensimon, Phys. Rev. A 30, 2840 (1984).

[19] S. D. Howison, J. Fluid Mech. 167, 439 (1986).

[20] S. D. Howison, Eur. J. Appl. Math. 3, 209 (1992).

[21] R. Folch, E. Alvarez-Lacalle, J. Ortín, and J. Casademunt, Phys. Rev. E 80, 056305 (2009).

[22] E. Alvarez-Lacalle, J. Casademunt, and J. Eggers, Phys. Rev. E 80, 056306 (2009).

[23] A. Leshchiner, M. Thrasher, M. B. Mineev-Weinstein, and H. L. Swinney, Phys. Rev. E 81, 016206 (2010).

[24] A. I. Pesci, M. A. Porter, and R. E. Goldstein, Phys. Rev. E 68, 056305 (2003).

[25] L. J. Cummings, S. D. Howison, and J. R. King, Eur. J. Appl. Math. 10, 635 (1999).

[26] P. Gondret and M. Rabaud, Phys. Fluids 9, 3267 (1997).

[27] C. Ruyer-Quil, C. R. Acad. Sci., Ser. IIb: Mec., Phys., Chim., Astron. 329, 337 (2001).

[28] F. Plouraboué and E. J. Hinch, Phys. Fluids 14, 922 (2002).

[29] C. Chevalier, M. Ben Amar, D. Bonn, and A. Lindner, J. Fluid Mech. 552, 83 (2006). 